\title{
Adherencia al control nutricional en varones con patologías crónicas
}

\author{
Adherence to nutritional check-up \\ in men with chronic diseases
}

\begin{abstract}
Introduction: One of the goals presented by the Cardiovascular Health Program (CHP) in Chile is the reduction of risk factors associated with metabolic syndrome. A part of the monitoring is achieved through check-ups and inquiries carried out by a multidisciplinary team, in which a nutritionist takes part. Objective: Interpret the perception of male adults, patients of the Boca Sur Cesfam and users of CHP, on the factors promoting attendance to nutritional check-ups. Subjects and methods: Using an interpretative qualitative paradigm with a phenomenological approach, theoretical saturation was acomplished during the tenth interview to male adults who fulfiulled the selection criteria of the study. Results: It is interpreted that the interviewees have various motives and interests in attending nutritional check-ups of the CHP. The most prominent ones are taking care of their general health, and control of the underlying disease. Family, especially their partners; the relationship built between the nutritionist and the patient, strenghtened by communication and trust are found to have a positive effect on the patient attending regularly to check-ups. Conclusions: That adherence to nutritional check-ups is favored by motivated male adults who seek to improve their quality of life attending a nutritional consultation, usually encouraged by their families, especially their spouses or partners. Also, when the relationship established with the professional helping them is perceived as positive. Friends are not considered influential on the attendance and monitoring of their underlying disease. Key words: Males; quality of life; adherence to nutritional checkup; qualitative design.
\end{abstract}

Claudia Troncoso P. (1)

Natalia Contreras C. (2) Solange González C. (2)

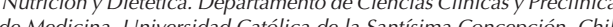
Facultad de Medicina. Universidad Católica de la Santísima Concepción, Chile. (2) Carrera de Nutrición y Dietética. Facultad de Medicina. Universidad Católica de la Santísima Concepción, Chile.

Dirigir la correspondencia a: Nutricionista Claudia Troncoso Pantoja Universidad Católica de la Santísima Concepción. Facultad de Medicina, Campus San Andrés. Alonso de Ribera 2850. Concepción, Chile.

Fonos: 56-041-2345427 - 56-041-2345406 Correo electrónico: ctroncosop@ucsc.cl

Este trabajo fue recibido el 21 de Julio de 2015 y aceptado para ser publicado el 20 de Noviembre de 2015.

dades (3-6).

En las personas con enfermedades crónicas, se identifican una serie de factores que influyen en la adherencia al tratamiento, resaltando: las familias, el acceso a los servicios de salud y el tipo y/o duración del tratamiento recibido. En general, los pacientes crónicos no presentan un adecuado seguimiento a las indicaciones médicas, debido a que tienden a no consumir sus fármacos o presentar una limitada asistencia a controles con el equipo de salud (7-9).

En Chile, el cuidado de personas con algún tipo de enfermedad crónica es identificado como un desafío para el sistema, en base a la necesidad de satisfacer las necesidades de estas personas, las que son variadas y complejas.

Para el año 2015, el Ministerio de Salud a través del Programa de Salud Cardiovascular (PSCV) presenta como una de las metas para los usuarios adultos, el contribuir a la 
disminución de los factores de riesgo cardiovascular asociados al síndrome metabólico, desarrollando parte de su seguimiento a través de controles y consultas realizadas por un equipo multidisciplinario, en el que participan el médico, la enfermera y la nutricionista, entre otros profesionales. Sobre los nutricionistas, las Estrategias de Intervención 2015 consideran la atención de este profesional a través de controles en el mes 1 , $3,6,9$ y 12 desde el ingreso de nuevos usuarios al Programa Vida Sana (10-13).

Frente a la limitada asistencia que los varones adultos presentan a sus controles de salud (14) y el reducido abordaje que se realiza en personas del género masculino en investigaciones temáticas, además de los esfuerzos que se están realizando a través de políticas públicas en el país orientadas al cuidado de estos usuario, surge esta investigación, que presenta como objetivo interpretar la percepción que presentan usuarios adultos de sexo masculino sobre los factores que favorecen la asistencia al control por nutricional del Cesfam Boca Sur, comuna de San Pedro de la Paz, Región del Bíobío, Chile durante el año 2015.

\section{SUJETOS Y MÉTODOS}

En esta investigación se utilizó el paradigma cualitativo interpretativo con enfoque fenomenológico para profundizar en la percepción sobre los factores que favorecen la asistencia al control nutricional de hombres adultos, referidos a algunos aspectos psicológicos como es la motivación y autoestima de los usuarios; el entorno social, específicamente las influencias de su familia, amigos y trabajo; la relación entre la nutricionista y el paciente y los factores asociados al tratamiento, profundizado en la adherencia a la dietoterapia y el tiempo demandado en la asistencia a controles.

El estudio se desarrolló en hombres de 35 a 64 años de edad, usuarios externos del Cesfam Boca Sur, comuna de San Pedro de la Paz, región de Bíobío durante el mes de marzo de 2015. Los criterios de selección del estudio incluían, además de la edad de los varones, pertenecer al Programa de Salud Cardiovascular (PSCV) y ser asistente a control por nutricionista por dos o más veces durante los meses de agosto de 2014 y marzo 2015 (se excluyeron varones de otros rangos etarios, con problemas cognitivos o de movilización, además de presentarse sin asistencia al control nutricional antes de agosto del año 2014). A lo anterior, se solicitó la firma del consentimiento informado. Esta última actividad fue la forma en que se controló los aspectos éticos en esta investigación, además de la presentación y autorización emanada de las autoridades del Centro de Salud a los investigadores antes de su realización. En el consentimiento informado, se les informa a los participantes de los objetivos del estudio, sobre la recolección de la información y la utilización que se le daría a esta última. Se garantizó la privacidad de la información y por sobre todo, resaltar que la participación es voluntaria y sin incentivos.

Para recolectar la información, se utilizó una entrevista semiestructurada, instrumento elaborado por investigadores y sometido a evaluación por expertos, además de prueba piloto antes de su aplicación.

La entrevista, realizada por los coautores capacitados para esta actividad, se llevó a cabo en dependencias del centro de salud (previa citación y confirmación telefónica) o en el domicilio del usuario, situación que ocurre para dos de los sujetos que participaron en el estudio. Las conversaciones personales fueron registradas en equipo de audio y/o celular, para ser transcritas y posteriormente analizadas.
El muestreo fue de tipo no probabilístico por conveniencia y el tamaño muestral se obtiene a través del punto de saturación teórico, esto es, la inexistencia de nuevos aportes de información proveniente de las entrevistas realizadas (15). Para esta investigación, la saturación se logra en la décima entrevista.

La técnica de análisis empleada para este estudio fue el análisis de contenidos. Para fortalecer la validez y confiabilidad de los resultados, se realiza una triangulación de informantes, en el en el que además de la participación de parte de los investigadores principales, se incorpora un antropólogo.

\section{RESULTADOS}

Los resultados se obtuvieron de diez entrevistas, momento en el cual se presenta la saturación de la información recolectada.

\section{Motivación y autoestima en la adherencia} a control nutricional del PSCV

Se interpreta que la totalidad de los sujetos entrevistados presentan diversas motivaciones e intereses en asistir a los controles nutricionales del PSCV, en el que predomina el cuidado de su estado de salud y el control de su patología de base, a través del apoyo profesional del nutricionista que los atiende.

Los usuarios entrevistados identifican cierta preocupación y temor de convivir con enfermedades crónicas al percibir, como parte del conocimiento colectivo, los efectos negativos de estas patologías. Además, se menciona el desconocimiento del tratamiento adecuado en forma rigurosa y que su estado de salud puede ser mejorado por la asistencia especializada del profesional afín.

Otra motivación en la asistencia a la consulta nutricional de la mayoría de los entrevistados era el interés y la preocupación por bajar de peso, ya que estaban conscientes de las consecuencias que la malnutrición por exceso provoca en el desarrollo o complicación de su enfermedad. En relación a esto último, les interesa asistir a los controles con el nutricionista porque pueden regular su alimentación, los alimentos a consumir y cuales deben restringir para lograr cambios positivos que favorezcan la disminución del peso corporal. Algunos sujetos de estudio manifestaron haber logrado notorias reducciones de peso, por motivación propia, pero principalmente por el apoyo del profesional, que le establece metas medibles, observables y realistas en cada control, ya que estas personas, si no perciben un reforzamiento externo sobre los riesgos o los estados de avances, estos les resultan más lejanas o inalcanzables.

En cuanto a la autoestima, se identificó una gran relación con la preocupación y valoración de sí mismos, principalmente en el cuidado de su salud y de su cuerpo, lo que puede traer mejorías a su vida y a la calidad de esta. La mayoría de los entrevistados manifestaron cuidar de su salud en mayor o menor grado mediante la alimentación y/o el desarrollo de actividad física, mientras que una minoría identificó un limitado interés por su autocuidado.

Los usuarios que percibían la necesidad de mantener un cuidado especial por su estado de salud, expresan que si bien, no logran tener un control óptimo de su alimentación o cumplir a cabalidad las metas del profesional que los atienden, están consientes de la importancia que conlleva el cambio de hábitos en estilos de vida, aunque estos últimos sean limitados, debido a la situación económica, el tiempo o el tipo de trabajo que presente la persona, que es percibida como una imposibilidad para realizar las indicaciones entregadas por el nutricionista.

Se identificó en la mayoría de los participantes, la pre- 
ocupación y el esfuerzo por querer hacer modificaciones en sus hábitos del cuidado en su salud, a través de cambios en su estilo de vida, originado, por ejemplo, por una situación aguda de complicación de su patología de base.

\section{Factores del entorno social que condicionan la} adherencia a controles nutricionales del PSCV

En esta investigación, el entorno social se enmarcó en la familia, amistades y en sus relaciones de amistades forjadas en el trabajo.

La mayoría de los pacientes entrevistados viven con su familia, conformada por esposa o pareja, hijos y/o nietos o padres. Estos perciben la preocupación y el apoyo por parte de esta en cuanto a su salud, reflejada en la preocupación que presentan frente al cuidado de su enfermedad y en la asistencia regular a los controles nutricionales del PSCV, además de presentar un rol esencial en el cuidado de la alimentación. Los usuarios entrevistados identifican que en este cuidado participan mayormente esposas e hijos/as, pero son las primeras quienes adquieren un papel fundamental, preparando y esforzándose en que los alimentos sean sanos y adecuados a la patología que ello presentan, y también recordándoles las indicaciones del nutricionista y asistiendo al centro de salud para obtener citación con el profesional. Los entrevistados codificaron, que son las parejas o esposas el gran apoyo con el que cuentan.

Los entrevistados que vivían con solo un miembro de su familia o solos, de igual manera sienten un apoyo y preocupación de sus familiares más cercanos.

La mayoría de los participantes refiere tener amigos y vecinos, ya sea en el sector donde viven o vivían anteriormente. A pesar de reconocer este vínculo, expresaron que estos no están informados de su enfermedad, que no reciben apoyo de su parte y por lo mismo, no perciben preocupación. Mencionan que no es tema de conversación entre ellos, desconocen sobre sus controles con nutricionista del PSCV, lo que evidencia que no existe influencia de esas personas en que los sujetos adhieran a los controles nutricionales.

Sólo un sujeto entrevistado afirmó tener una buena relación con sus vecinos y comentan atenderse con la misma profesional, lo que implícitamente deja ver un apoyo entre ellos en cuanto a conocimientos de la enfermedad y de los controles nutricionales, que finalmente puede traducirse en un apoyo para que el paciente asista a los controles.

La mayoría de los entrevistados se encontraba con trabajo estable. Con respecto a las relaciones sociales dentro del trabajo, los sujetos afirman explícitamente que sus compañeros de trabajo no influyen en el control de su patología de base y su asistencia a los controles nutricionales del programa, principalmente porque en el campo laboral, sus pares desconocen de su enfermedad.

Sólo algunos sujetos manifestaron trabajar con familiares. En esta situación, se invierte la condición mencionada, ya que sus empleadores o compañeros de trabajo están al tanto de su enfermedad y por lo tanto, perciben un apoyo de parte de estos. De los empleadores, los entrevistados perciben cierto canalizado en permisos o disponibilidad horaria para asistir al CESFAM. Plantean la preocupación de sus empleadores y compañeros aludiendo a que les inducen a asistir a estos últimos.

\section{Relación profesional paciente en la adherencia a controles nutricionales del PSCV}

Entre los factores de la relación nutricionista paciente que condicionan la adherencia a controles, en esta investigación se profundizó en la comunicación, confianza y credibilidad que se crea en este binomio.

En relación a la comunicación existente el entre profesional y el paciente dentro del control nutricional, la mayoría de los entrevistados afirmaron que lo planteado por el nutricionista es realizado de manera clara y de fácil comprensión. Refieren que frente a cierta incertidumbre durante el control, ya sea por falta de información o por la complejidad de su enfermedad, tienen la capacidad de plantearlas al profesional ya que estas son contestadas en un lenguaje comprendido por el usuario y replicado cada vez que fuera necesario, reconociendo del profesional una actitud colaboradora y gentil.

En relación a la segunda categoría, casi la totalidad los entrevistados (nueve) relatan presentar la confianza suficiente para plantear sus inquietudes ante el profesional. Algunos refieren manifestarse en forma explícita cuando presentan desacuerdo ante indicaciones o recomendaciones dadas en el control, asimismo comparten sus inquietudes, temores y sugerencias.

Otros mencionan que plantean sus dudas con el profesional, pero no precisamente sus inquietudes respecto a la enfermedad y su actual condición, percibiendo que se requiere de una relación más cercana para lograr este nivel de confianza.

Algunos entrevistados indican no haber generado una relación de confianza con el nutricionista, principalmente porque sus controles no fueron realizados por el mismo profesional, no obstante, no influyó en su adherencia a los controles, ya que asisten cada vez que son citados al CESFAM.

En cuanto a la credibilidad del nutricionista que los atiende, con respecto a la seguridad, seriedad y objetividad que presenta como profesional, la totalidad de los entrevistados expresan respuestas positivas. Los sujetos identifican la objetividad de las nutricionistas que los atienden, identificando que actúan con seguridad en relación a los conocimientos de las enfermedades que presentan y percibiendo credibilidad desde un punto de vista profesional.

Se plantean, dos lineamientos. El primero en relación con algunos pacientes que mencionan recibir una buena indicación, sin embargo, el obtener resultados no depende de cómo se les entregue la información, sino de su interés por seguir dichas indicaciones.

Con respecto al segundo lineamiento, se identifica que las indicaciones entregadas por el profesional no están dentro del nivel socioeconómico de algunos sujetos, lo que influye directamente en el incumplimiento de sus metas nutricionales y de control metabólico. Algunos entrevistados expresan que los cambios solicitados son, en su mayoría, a corto plazo, lo que es codificado como una limitante al momento de realizar su tratamiento, sin embargo, esta situación no es un factor que limite su asistencia a los controles nutricionales.

\section{Factores asociados al tratamiento que condicionan la} adherencia a controles nutricionales del PSCV

Entre de los factores asociados al tratamiento, se identificaron las categorías dietoterapia y tiempo demandado en la asistencia a controles.

En cuanto a la dieta como parte de la terapia, la mayoría de los entrevistados relata que las recomendaciones e indicaciones dadas por el nutricionista conllevan mejorías a su estado de salud, percibiendo que es necesario incorporarlas a su vida diaria. Identifican que no habrá recuperación total de su patología de base, pero si hay convencimiento de que mediante la optimización de su calidad de vida se puede 
prolongar la misma. Asimismo, algunos plantean que estas mejorías se van a conseguir en la medida que ellos cumplan las indicaciones entregadas.

Otros expresan la dificultad para seguir la dietoterapia de manera estricta, ya que muchas veces las indicaciones dadas por el profesional no son realistas a la condición general del entrevistado, en especial, en el aspecto económico y su dificultad en la adquisición de ciertos alimentos que son recomendados para su dieta.

El total de los entrevistados reconoce que la dietoterapia realizada por la profesional ha traído mejorías para su salud e identifican que seguirá favoreciendo en el futuro, lo que los motiva a mantener sus controles nutricionales.

En relación al tiempo demandado que conlleva la asistencia a los controles nutricionales del PSCV, los sujetos entrevistados en su totalidad consideraron como un tiempo provechoso y no perdido. Sin embargo, son diversos los motivos que perciben para esta asistencia: algunos buscan mejorar su calidad de vida y tener un mayor bienestar, así como aumentar su conocimiento sobre su enfermedad y tratamiento o una búsqueda de un profesional que los apoye en lograr un mayor control y manejo de su enfermedad.

\section{DISCUSIÓN}

En el artículo de Ledón (16), se identifica que las personas con alguna patología crónica son los actores claves para potenciar su estado de salud, su propio bienestar y también una adecuada calidad de vida. Similar a este resultado, los usuarios entrevistados refieren que el fin último percibido como esencial en la asistencia a control con nutricionista es mejorar y/o mantener su calidad de vida y salud.

En esta investigación, las personas estudiadas percibían la necesidad de cuidarse y mantener un adecuado control de su patología crónica y adherencia a su tratamiento, sin embargo, identifican un conocimiento limitado de su enfermedad, lo que es codificado como un factor negativo para su control y adherencia. Esta situación es identificada en otros estudios, en el que los sujetos participantes y en algunos casos, las personas que les rodean, presentan escasos conocimientos de la enfermedad y por lo tanto un inadecuado control y/o adherencia al tratamiento (17-18). El estudio realizado por Soria et al (19) en personas con patologías crónicas, concluye que los pacientes que reciben información sobre su tratamiento son más adherentes que cuando un miembro del equipo de salud les explica sobre su enfermedad.

Urzúa (20) identifica que a mayor autoestima de la persona con alguna patología crónica, mayor será la calidad de vida reportada. Nuestros entrevistados identifican que entre sus motivaciones en la asistencia a control, están el cuidado de su salud y de su cuerpo, lo que relacionan con mejorías a su calidad de vida.

Existe una relación entre el exceso de peso corporal y las complicaciones inherentes a las patologías crónicas de adultos (21-23). Los participantes en nuestro estudio, percibían las consecuencias que la malnutrición por exceso provoca y que condiciona el control de su enfermedad. Por este motivo, les interesa asistir a los controles con el nutricionista, ya que identifican que al regular su alimentación, pueden lograr cambios positivos que favorezcan la disminución del peso corporal y por lo tanto, el control de su patología crónica.

En cuanto a los condicionantes percibidos del entorno social, la familia es identificada como esencial para los entrevistados en el seguimiento del tratamiento y la asistencia al control por parte del profesional del área de salud. Esta situación se replica en los estudios realizados por Iglesias et al, Urzúa et al y por Vega et al (24-26). Este último identifica a la familia como el centro de apoyo social que presentan personas con enfermedades crónicas para afrontar exitosamente las complicaciones de su patología de base. A lo anterior, ambos autores coinciden con los resultados de nuestro estudio.

En el grupo familiar, la pareja o esposa son percibidos como apoyo fundamental en la adherencia a su tratamiento, por diversas motivaciones, como lo son la solicitud de citación para controles o porque preparan sus alimentos. Esta situación coincide con lo encontrado por Soria et al (27), que profundiza en la imagen cultural que se asigna a las mujeres, responsable de gran parte de la carga doméstica, incluyendo el cuidado de los miembros de la familia que estén con algún tipo de patología.

Los entrevistados identificaron presentar amistades en diversos ámbitos, destacando el trabajo o lugar de residencia, pero sin embargo, estos últimos no son percibidos como relevantes en la adherencia a su tratamiento, ya que su patología de base y por lo tanto su control, no es tratado en su relación de amistad, identificando la falta de interés, tanto de los pacientes con alguna patología (al no mencionar sobre esta última y su tratamiento) y de sus amistades, que no se informan sobre la condición de salud de estas personas. Esta situación se contrapone a lo encontrado por Gómez et al (28), que identifica que el apoyo social, entre otros de vecinos y amigos, sería una estrategia de afrontamiento necesaria en el curso de la enfermedad.

En cuanto a la relación que se establece entre el profesional de salud y el paciente, estudios identifican una relación positiva, lo que significa para la persona atendida el percibir que recibe un mejor control, sentirse valorado por el equipo médico, comprender su enfermedad o usar estrategias para mejorar la adherencia a su tratamiento y visitas al equipo de salud (29-31). Los entrevistados de nuestra investigación, identifican una comunicación positiva con el profesional que los atiende, los que les da credibilidad y favorecen el control y adherencia a la asistencia a estos. La mayoría de los usuarios presentan cierta confianza con el profesional que los atiende, permitiendo dirigirse y plantear diversas inquietudes, interpretando positivamente que la comunicación establecida con el profesional, es beneficioso para su calidad de vida, permitiéndoles adquirir o profundizar sus conocimientos sobre su enfermedad y su tratamiento dietético, apreciando resolver sus inquietudes de manera sencilla y comprensible.

El nutricionista presenta entre sus competencias asistenciales el desarrollo de educación individual y familiar para proporcionar atención dietética y dietoterapéutica a través del ciclo vital (32-34). En nuestro estudio, los usuarios refieren la importancia del nutricionista en la adherencia al tratamiento, a través de prescripción dietética y la percepción de los beneficios que conlleva en su calidad de vida y salud.

Oliva et al (35) identificaron la empatía que presenta el nutricionista al desarrollar su consulta y su capacidad en la entrega de un tratamiento de calidad, con intervenciones efectivas para el logro de sus objetivos en la consulta nutricional. Esta última situación no es compartida por todos los entrevistados, mencionando que las recomendaciones dietéticas, no siempre responden a su actual situación de vida, en especial por aspectos económicos, lo que les dificulta realizar una dieta acorde a la indicación entregada por el profesional. Esto último es importante, ya que por diversas circunstancias, la presión asistencial, el nutricionista y otros profesionales de la salud, entregan sus indicaciones sin condicionar o profundizar 
en la realidad de la persona que se está atendiendo.

Una de las limitantes de este estudio fue la dificultad para los investigadores en la realización de las entrevistas, debido a la complicación que surgió por los horarios de trabajo que presentaban los sujetos seleccionados y la dificultad de coordinar los tiempos para la recolección de información.

En general, los adultos varones no son un grupo etario de preferencia en el desarrollo de distintas investigaciones en la temática de las patologías crónicas. Llamó la atención, siendo un hallazgo en este estudio, el desinterés que los varones presentaban en compartir aspectos de su condición patológica con sus amistades, los que pueden ser consideradas entre las redes de apoyo que pueden fortalecer su adherencia al control y tratamiento, además de la asistencia a la consulta nutricional o de otro profesional del área de salud.

\section{CONCLUSIONES}

La adherencia que presentan varones adultos pertenecientes al Programa de Salud Cardiovascular para asistir a sus controles con nutricionista, se deben a motivaciones internas, destacando la percepción de la importancia en mejorar o mantener una adecuada calidad de vida y salud. La familia, en especial la pareja o esposa, refuerza positivamente su asistencia al control nutricional.

Los entrevistados identifican como un factor favorable para la asistencia al control y adherencia al tratamiento de su cuadro de base, la comunicación que se establece con el profesional, lo que permite fortalecer los lazos de confianza y credibilidad entre nutricionista y paciente, que a largo plazo, es percibido como un incentivo para un mejor estado de salud.

El tiempo invertido para asistir a sus controles, es considerado como provechoso para mejorar su estado de salud, al igual que las indicaciones generales y dietoterapéuticas que son entregadas en estas consultas nutricionales, aunque para algunos entrevistados, estas últimas no están adaptadas a su realidad económica.

El círculo de amistades en general, incluyendo el de su ámbito laboral, no condiciona la adherencia al control por nutricionista.

\section{RESUMEN}

Introducción: Entre las metas que presenta el Programa de Salud Cardiovascular (PSCV) en Chile están la disminución de los factores de riesgo asociados al síndrome metabólico. Parte de su seguimiento se realiza a través de controles y consultas realizadas por un equipo multidisciplinario, en el que participa el nutricionista. Objetivo: Interpretar la percepción de adultos de sexo masculino, atendidos en el Cesfam Boca Sur y usuarios del PSCV, sobre los factores que favorecen la concurrencia al control por nutricionista. Sujetos y métodos: Utilizando el paradigma cualitativo interpretativo con enfoque fenomenológico, se logra el punto de saturación del estudio en la décima entrevista de varones adultos que cumplieron con los criterios de selección del estudio. Resultados: Se interpreta que los sujetos entrevistados presentan diversas motivaciones e intereses en asistir a los controles nutricionales del PSCV, en el que predomina el cuidado de su estado de salud y el control de su patología de base. La familia, en especial la pareja, la relación que se establece entre el nutricionista y el paciente, fortalecido esto último por la comunicación y confianza que se estable, actúa positivamente en la asistencia a la consulta en forma regular. Conclusión: Los varones adultos que asisten a una consulta nutricional, están motivados en mejorar su calidad de vida, siendo apoyados por la familia en especial por su esposa o pareja estable y percibiendo positivamente la relación que se forja con el profesional que lo atiende, lo que favorece la adherencia a sus controles. Los amigos no son considerados como influyentes en la asistencia y el control de su patología de base.

Palabras clave: Varones; calidad de vida; adherencia a control; diseño cualitativo.

\section{BIBLIOGRAFÍA}

1. Martin L, Bayarre H, Grau J. Validación del cuestionario MBG (Martín-Bayarre-Grau) para evaluar la adherencia terapéutica en hipertensión arterial. Rev Cubana Salud Pública. 2008; 34(1): 0-0.

2. Silva G, Correa J. Adherencia al tratamiento. Acta Médica Colombiana. 2008; 30(4): 268-273.

3. Ortiz M, Ortiz E. Psicología de la salud: una clave para comprender el fenómeno de la adherencia terapéutica. Rev Méd Chile. 2007; 135(5): 647-52.

4. Zambrano R, Duitama J, Posada J et al. Percepción de la adherencia a tratamientos en pacientes con factores de riesgo cardiovascular. Rev Fac Nac Salud Pública. 2012; 30(32):163-74.

5. Dilla T, Valladares A, Lizán L et al. Adherencia y persistencia terapéutica: causas, consecuencias y estrategias de mejora. Aten Primaria. 2009; 41(6): 342-8.

6. Escamilla J, Castañer O, Benito $S$ et al. Motivos de incumplimiento terapéutico en pacientes mayores polimedicados, un estudio mediante grupos focales. Aten Primaria. 2008; 40(2): 81-5.

7. Soria $R$, Vega $Z$, Nava $C$ et al. Interacción médico-paciente y su relación con el control del padecimiento en enfermos crónicos. Liberabit: Lima (Perú). 2011; 17(2): 223-30.

8. Alayón A, Altamar D, Banquez C et al. Complicaciones crónicas, hipertensión y obesidad en pacientes diabéticos en Cartagena, Colombia. Rev Salud Pública. 2009; 11(6): 857-64.

9. Llisterri J, Rodríguez G, Alonso F et al. Aportaciones del estudio PRESCAP al conocimiento de la hipertensión en España. SEMERGEN. 2009; 35(9): 450-6.

10. Minsal. Modelos de Atención para personas con enfermedades crónicas. Directrices para la implementación. Disponible en WWW: http://wWw.redcronicas. cl/wrdprss_minsal/wp-content/uploads/2015/03/ MODELO-DE-ATENCION-PARA-PERSONAS-CONENFERMEDADESCR\%C3\%93NICAS_final_web.pdf Obtenido en junio 2015.

11. Minsal. ¿Cómo integrar promoción en otros Programas? La visión desde el Programa de Salud Cardiovascular. Disponible en WWW: http://web.minsal.cl/sites/default/ files/Promoci\%C3\%B3n_Salud_PSCV.pdf Obtenido en junio 2015.

12. Minsal. Orientaciones para la Planificación y programación en Red año 2015. Disponible en WWW: http://web. minsal.cl/sites/default/files/Orientaciones_red_2015.pdf Obtenido en junio 2015.

13. Minsal. Programa Vida Sana: Intervención en Factores de Riesgo de Enfermedades no Trasmisibles. 2015. Disponible en WWW: http://WwW.redcronicas.cl/temas-de-salud/ salud-cardiovascular-2/enfermedades-cardiovasculares/ programa-de-salud-cardiovascular/jornada-nacionalpscv-2015/ Obtenido en junio 2015.

14. Rosa-Jiménez F, Montijano A, Herráiz C et al. ¿Solicitan las mujeres más consultas al área médica que los hombres? An Med Interna. 2005; 22(11): 515-9. 
15. Osses S, Sánchez I, Ibañez F. Investigación cualitativa en educación. Hacia la generación de teoría a través del proceso analítico. Estud Pedagog. 2006; 32:119-33.

16. Ledón L. Enfermedades crónicas y vida cotidiana. Rev Cubana Salud Pública. 2011; 37(4): 488-99.

17. Bustos $R$, Barajas $A$, López $G$ et al. Conocimientos sobre diabetes mellitus en pacientes diabéticos tipo 2 tanto urbanos como rurales del occidente de México. Arch Med Fam. 2007; 9(3): 147-59.

18. Pinzón M, Aponte L, Hernández R. Experiencias de los cuidadores informales en el manejo de la diabetes mellitus tipo II. ORINOQUIA. 2013; 17(2): 241-51.

19. Soria $R$, Vega $Z, N a v a C$ et al. Interacción médico-paciente y su relación con el control del padecimiento en enfermos crónicos. Liberabit. 2011; 17(2): 223-30.

20. Urzúa A. Calidad de vida y factores biopsicosociales en patologías médicas crónicas. Ter Psicol. 2008; 26(2): 207-14.

21. Falgade M, Del Solar J, Gerrero $M$ et al. Factores de riesgo de enfermedades crónicas no trasmisibles en funcionarios de una empresa de servicios financieros en la Región Metropolitana. Rev Méd Chile. 2005; 133(8): 919:28.

22. Salinas J, lera L, González C et al. Estilos de vida, alimentación y estado nutricional en trabajadores de la construcción de la Región Metropolitana de Chile. Rev Méd Chil. 2014; 142(7): 833-40.

23. Ratner $R$, Sabal J, Hernández $P$ et al. Estilos de vida $y$ estado nutricional de trabajadores en empresas públicas y privadas de dos regiones de Chile. Rev Méd Chil. 2008; 136(11): 1406-14.

24. Iglesias I, Castelo L, Domínguez E et al. Adherencia terapéutica en pacientes con dislipoproteinemias. Rev Cubana Endocrinol. 2013; 24(3): 229-41.

25. Urzúa A, Chirino A, Valladares G. Autoreporte de la calidad de vida relacionada con la salud en diabetes mellitus tipo 2. Rev Med Chil. 2011; 139(3): 313:20.

26. Vega O, González D. Apoyo Social: elemento clave en el afrontamiento de la enfermedad crónica. Enferm Glob. 2009; 16:1-11.

27. Soria $R$, Ávila $D$, Vega $Z$ et al. Estrés familiar y adherencia terapéutica en pacientes con enfermedades crónicas. Alter Psicol. 2012; 16(26): 78-84.

28. Gómez M, Lagoueyte M. El apoyo social: estrategia para afrontar en cáncer de cérvix. Av Enferm. 2012; 30(1): 32-41.

29. Azcarate E, Hernández I, Guzmán M. Percepción en la calidad de la relación médico - paciente en casos de hipertensión arterial sistémica. Aten Fam. 2014; 21(3): 83-5.

30. Prieto $M$, Danet $A$, Escudero $M$ et al. Definición de competencia médica según pacientes crónicos el sistema sanitario público de Andalucía. Gac Sanit. 26(5): 450-6.

31. Martínez J, Villa J, Quintero A et al. Frecuencia de factores de riesgo cardiovascular en pacientes hipertensos en un hospital de segundo nivel. Rev Fac Nac Salud Pública. 2011; 29(2): 139-44.

32. Labraña A, Durán E, Soto DCompetencias el Nutricionista en el área de Atención Primaria de Salud. Rev Chil Nutr. 2005; 32(3): 239-46.

33. Labraña A, Durán E, Asenjo $G$ et al. Plan de estudios basados en competencias para la carrera de Nutrición y Dietética de la Universidad de Concepción. Rev Chil Nutr. 2010; 37(3): 302-7.

34. González A, Bell Z, Pérez $M$ et al. Sobre las competencias de los Nutricionistas en los ámbitos hospitalarios y comunitarios. Rev Cubana Aliment Nutr. 2011; 21(1): 159-74.

35. Oliva $P$, Buhring $K$, Godoy $S$ et al. Percepción de la función profesional del Nutricionista por parte de los usuarios de atención primaria. Rev Chil Nutr. 2010; 37(2): 165-8. 